\title{
MicroRNA-490-3p suppresses the proliferation and invasion of hepatocellular carcinoma cells via targeting TMOD3
}

\author{
HONGHUA WANG, GUOBAO YANG, YANG YU and PEIBING GU \\ Department of General Surgery, Xiangshui County People's Hospital, Yancheng, Jiangsu 224600, P.R. China
}

Received December 3, 2019; Accepted July 22, 2020

DOI: 10.3892/ol.2020.11956

\begin{abstract}
Hepatocellular carcinoma (HCC) is a primary malignancy of the liver. It has been reported that microRNAs (miRs) play important roles in the progression and development of HCC. The expression of miR-490-3p has been shown to be downregulated in HCC tissues. Therefore, the present study aimed to investigate the effects of miR-490-3p on HCC cells and the underlying mechanism. Cell Counting Kit-8, flow cytometry, and Transwell migration and invasion assays were performed to determine the viability, apoptosis, migration and invasion of HCC cells, respectively. Furthermore, a luciferase activity assay was used to verify the association between miR-490-3p and its predicted target tropomodulin 3 (TMOD3). In addition, the protein levels of Bax, Bcl-2, cleaved caspase-3, TMOD3, phosphorylated (p)-p38 and p-ERK in HCC cells were detected using western blot analysis. The results demonstrated that the overexpression of miR-490-3p via transfection with miR-490-3p mimics significantly inhibited the proliferation of Huh-7 and HEP 3B2.1-7 cells. In addition, overexpression of miR-490-3p markedly suppressed the migration and invasion abilities of Huh-7 cells. miR-490-3p mimics significantly induced liver cancer cell apoptosis via upregulating Bax and cleaved caspase- 3 and downregulating anti-apoptotic protein Bcl-2. Additionally, a luciferase activity assay indicated that TMOD3 is a downstream target gene of miR-490-3p. The protein levels of TMOD3, p-p38 and p-ERK were significantly downregulated in Huh-7 cells following transfection with miR-490-3p mimics, and the overexpression of TMOD3 attenuated these effects. In conclusion, the aforementioned results suggest that the overexpression of miR-490-3p inhibited the proliferation and invasion of HCC cells by targeting TMOD3. Therefore, the miR-490-3p/TMOD3 axis may be a potent target for the treatment of HCC.
\end{abstract}

Correspondence to: Dr Guobao Yang, Department of General Surgery, Xiangshui County People's Hospital, 94 Guanhe Road, Yancheng, Jiangsu 224600, P.R. China

E-mail: yangubao1446@163.com

Key words: miR-490-3p, hepatocellular carcinoma, TMOD3, apoptosis

\section{Introduction}

Liver cancer is one of the most common malignant tumors with high incidence and mortality, which are ranked sixth and third worldwide, respectively, according to the GLOBOCAN estimates published in 2018 (1). Hepatocellular carcinoma (HCC) accounts for $>90 \%$ of all primary liver cancers and occurs predominantly in patients with underlying chronic liver disease triggered by host and environmental factors (2). For example, hepatocellular adenoma (HCA), a rare benign tumor, possesses a high risk of transformation into HCC (3). Several therapeutic regimens and drugs, including surgery, radiotherapy, chemotherapy as well as traditional Chinese medicine have been applied at different stages of HCC to increase the survival rates of patients and improve their quality of life. However, as patients with HCC are usually asymptomatic during the early stages of the disease and the progression of $\mathrm{HCC}$ is rapid, the majority of patients are incidentally diagnosed at an advanced stage (4). In addition, the present treatment strategies for advanced and unresectable HCC require further development. Therefore, it is necessary to identify novel therapeutic targets for the treatment of patients with HCC.

MicroRNAs (miRNAs, miRs) are a class of non-coding RNAs $\sim 22$ nucleotides in length that regulate posttranscriptional gene expression (5) and are widely involved in various processes of carcinogenesis. It is now generally accepted that the dysregulation of miRNAs with tumor-suppressive or oncogenic functions may affect the development of tumors (6). Li et al (7) demonstrated that miR-34a acted as a tumor suppressor in breast cancer via the downregulation of Bcl-2. In addition, another study revealed that increased miR-21 expression was associated with poor prognosis in patients with breast cancer, indicating that miR-21 acts as an oncogene in breast cancer (8). Therefore, miRNAs may serve as promising biomarkers in the diagnosis, recurrence and prognosis of human cancers.

There is considerable evidence that miRNAs play important roles in the development and progression of HCC (9). It has been reported that miR-221 is markedly upregulated in HCC cells, and its overexpression promotes the proliferation and invasion of HCC cells (10,11). Furthermore, Zheng et al (12) demonstrated that the expression levels of miR-490-3p were significantly lower in HCC and HCA tissues compared with normal liver tissues. However, the role of miR-490-3p in 
HCC has not yet been fully elucidated. Therefore, the present study aimed to investigate the role of miR-490-3p in HCC tumorigenesis, which may suggest a novel treatment strategy for HCC.

\section{Materials and methods}

Cell culture. Two human HCC cell lines, namely Huh-7 and HEP 3B2.1-7, were obtained from the American Type Culture Collection. MIHA immortalized human hepatocytes were purchased from Mingzhou Biotechnology Co., Ltd. (cat. no. MZ-1157). The cells were maintained in high-glucose Dulbecco's modified Eagle's medium (DMEM-H; Gibco; Thermo Fisher Scientific, Inc.) supplemented with $10 \%$ fetal bovine serum (FBS; Invitrogen; Thermo Fisher Scientific, Inc.), and $1 \%$ penicillin and streptomycin $(100 \mathrm{U} / \mathrm{ml})$. Cells were cultured at $37^{\circ} \mathrm{C}$ in an atmosphere of $5 \% \mathrm{CO}_{2}$ and $95 \%$ air.

Cell transfection and lentiviral infection. miRNA-490-3p mimics and negative control (NC) mimics were obtained from Shanghai GenePharma Co., Ltd.. Huh-7 and HEP 3B2.1-7 cells $\left(5 \times 10^{3} /\right.$ per well) were seeded onto 6 -well plates and cultured at $37^{\circ} \mathrm{C}$. When the cells reached $70-80 \%$ confluence, they were transfected with miR-490-3p or NC mimics $(10 \mathrm{nM})$ for $6 \mathrm{~h}$ using Lipofectamine 2000 (Thermo Fisher Scientific, Inc.) according to the manufacturer's protocol. Following transfection, the medium was replaced with fresh DMEM (Gibco; Thermo Fisher Scientific, Inc.) and the cells were incubated for an additional $42 \mathrm{~h}$ (For transwell assay, cells were incubated for an additional $18 \mathrm{~h}$ ). The sequences were as follows: miR-490-3p mimics, 3'-GUCGUACCUCAGGAGGUCCAAC-5'; NC mimics, 3'-GGGTTACGATTGCCCAGAT-5'.

The tropomodulin 3 (TMOD3) overexpression plasmid (pLVX-IRES-Puro-TMOD3) was purchased from Shanghai GenePharma Co., Ltd.. Subsequently, 293T cells (American Type Culture Collection) were infected with $1 \mu \mathrm{g} / \mu \mathrm{l} \mathrm{pLVX}$-IRES-Puro-TMOD3 or empty vector. Following infection for $72 \mathrm{~h}$, the lentiviral particles were collected and concentrated. Huh-7 cells of $60-80 \%$ confluence were seeded onto 6-well plates overnight prior to infection. Subsequently, cells were infected with the lentiviral particles for $24 \mathrm{~h}$ and then the infection medium was replaced with fresh medium. Puromycin $(2.5 \mu \mathrm{g} / \mathrm{ml}$; Thermo Fisher Scientific, Inc.) was added to the medium to select stable Huh-7 cells and $48 \mathrm{~h}$ following infection, cells were collected for subsequent experiments.

Reverse transcription-quantitative PCR (RT-qPCR). Total RNA was extracted from the cells using TRIzol reagent (Invitrogen; Thermo Fisher Scientific, Inc.) and cDNA was then synthesized with the PrimeScript ${ }^{\mathrm{TM}}$ RT reagent kit (Takara Biotechnology Co., Ltd.), according to the manufacturer's protocol. Subsequently, qPCR was performed using a SYBR ${ }^{\circledR}$ Premix Ex Taq ${ }^{\mathrm{TM}}$ kit (Takara Biotechnology Co., Ltd.) according to the manufacturer's instructions. All experiments were carried out in triplicate and the fold change of gene expression was calculated using the $2^{-\Delta \Delta \mathrm{Cq}}$ method as previously described (13). The primer sequences used were as follows: For miR-490-3p, forward, 5'-CAACCTGGAGGACTCCATGC-3' and reverse, 5'-CTCAACTGGTGTCGTGGAGTC-3'; for
U6, forward, 5'-CTCGCTTCGGCAGCACAT-3' and reverse, 5'-AACGCTTCACGAATTTGCGT-3'; for TMOD3, forward, 5'-CCACCAAATCCAACCAATGTAG-3' and reverse, 5'-TCA GTGCCAGAATCCCAACTC-3'; and for $\beta$-actin, forward, 5'-GTCCACCGCAAATGCTTCTA-3' and reverse, 5'-TGC TGTCACCTTCACCGTTC-3'. The RT-qPCR conditions used were as follows: $2 \mathrm{~min}$ at $94^{\circ} \mathrm{C}$, followed by 35 cycles (30 sec at $94^{\circ} \mathrm{C}$ and $45 \mathrm{sec}$ at $55^{\circ} \mathrm{C}$ ). U6 and $\beta$-actin were used as the internal controls for miR-490-3p and TMOD3, respectively.

Cell counting Kit-8 (CCK-8) assay. Cell proliferation was assessed using a CCK- 8 assay kit (Dojindo Molecular Technologies, Inc.) according to the manufacturer's protocol. Briefly, Huh-7 and HEP 3B2.1-7 cells were transfected with miR-490-3p or NC mimics for $0,24,48$ and $72 \mathrm{~h}$. Then, $10 \mu \mathrm{l}$ CCK-8 reagent was added to each well and cells were incubated for an additional $2 \mathrm{~h}$ prior measurement of the absorbance at $450 \mathrm{~nm}$.

Flow cytometric analysis. An Annexin V-FITC/PI Apoptosis kit (Thermo Fisher Scientific, Inc.) was used to stain apoptotic cells and distinguish them from normal cells in a subsequent analysis using a flow cytometer (FACScan; BD Biosciences). Briefly, Huh-7 and HEP 3B2.1-7 cells were washed three times with PBS (Thermo Fisher Scientific, Inc.) and then fixed with $100 \mu 1$ binding buffer (Thermo Fisher Scientific, Inc.). Subsequently, $5 \mu 1$ propidium iodide and $5 \mu 1$ Annexin V-FITC were used to stain the cells for $15 \mathrm{~min}$ in the dark. Finally, cells were analysed with a flow cytometer (Flowjo V10.6.2, BD Biosciences).

Western blot analysis. Total protein was extracted from cell lines using RIPA lysis buffer (Beyotime Institute of Biotechnology). Total protein concentration of the Huh-7 cell extract was determined using a BCA Protein Assay kit (Sigma-Aldrich; Merck KGaA). Subsequently, total proteins were separated by $10 \%$ sodium dodecyl sulfate polyacrylamide gel electrophoresis and then transferred onto polyvinylidene difluoride (Thermo Fisher Scientific, Inc.) membranes. Following blocking with 5\% fat-free milk at room temperature for $1 \mathrm{~h}$, the membranes were incubated with primary antibodies overnight at $4^{\circ} \mathrm{C}$ and then washed three times with TBS supplemented with Tween-20 followed by incubation with a horseradish peroxidase-conjugated secondary antibody (dilution, 1:5,000; cat. no. ab6721; Abcam) at room temperature for $1 \mathrm{~h}$. Subsequently, protein bands were visualized using an Enhanced Chemiluminescence Detection System (Thermo Fisher Scientific, Inc.). Primary antibodies used in the present study were as follows: Anti-Bax (dilution, 1:1,000; cat. no. ab32503), anti-caspase-3 (dilution, 1:1,000; cat. no. ab13847), anti-Bcl-2 (dilution, 1:1,000; cat. no. ab32124), anti-phosphorylated (p)-p38 (dilution, 1:1,000; cat. no. ab170099), anti-p-ERK (dilution, 1:1,000; cat. no. ab229912), anti-p-Akt (dilution, 1:1,000; cat. no. ab18785), anti-Akt (dilution, 1:1,000; cat. no. ab8805), anti-p38 (dilution, 1:1,000; cat. no. ab31828), anti-ERK (dilution, 1:1,000; cat. no. ab53277) and anti- $\beta$-actin (dilution, 1:1,000; cat. no. ab8226). All the antibodies were purchased from Abcam. IPP 6.0 (Image-Pro Plus 6.0) was used for the densitometry analysis. 
A

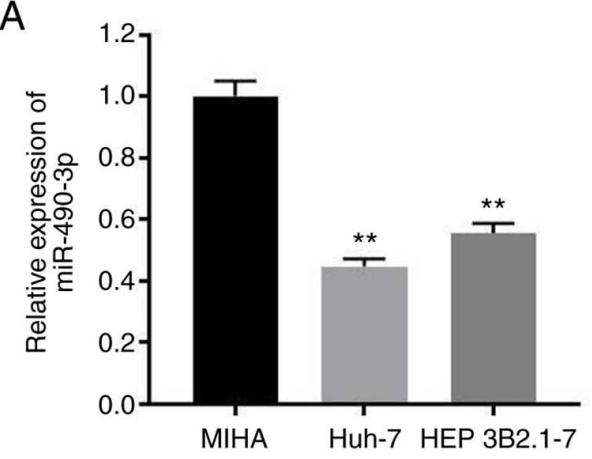

C

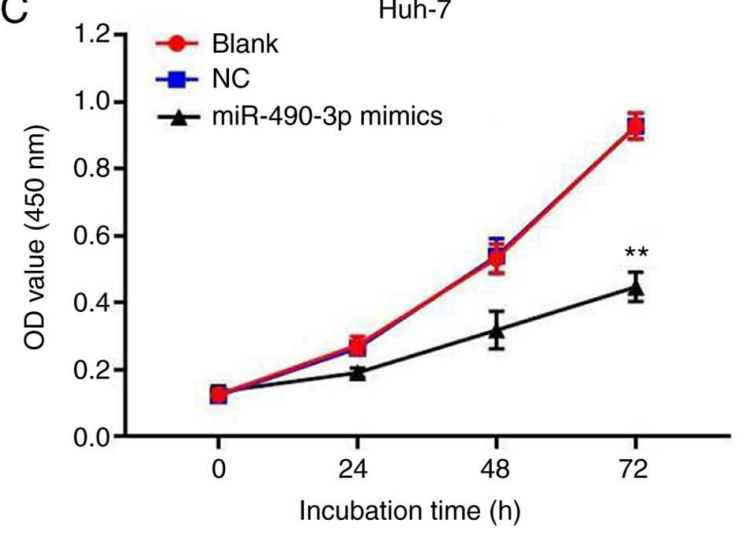

B

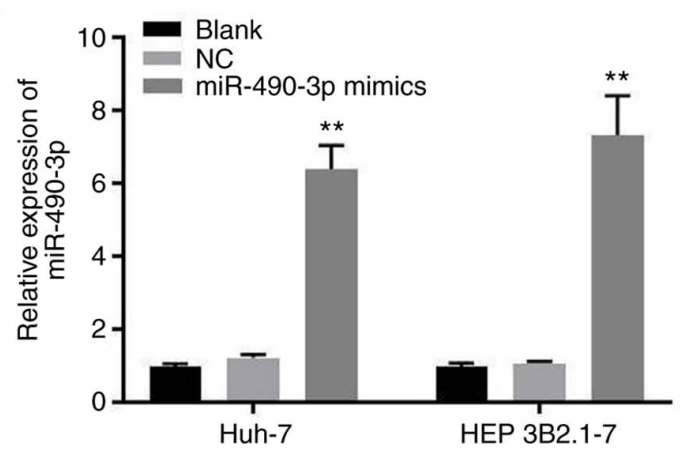

D

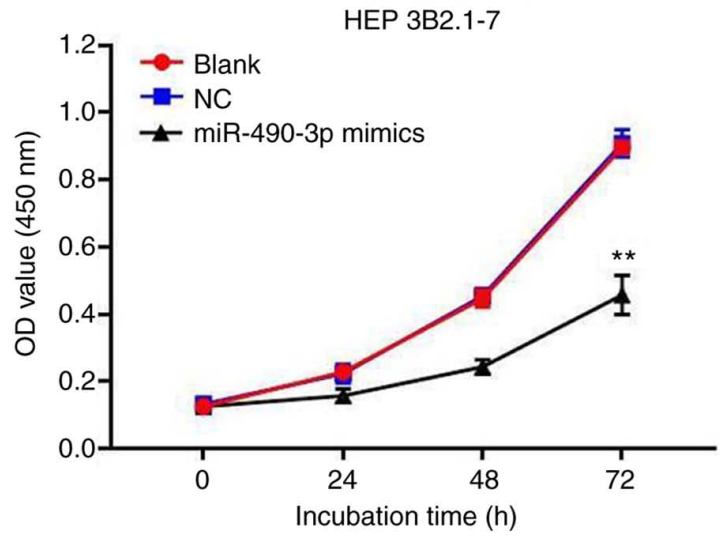

Figure 1. Overexpression of miR-490-3p inhibits the proliferation of HCC cells. (A) Levels of miR-490-3p in human hepatocytes and HCC cells were determined using RT-qPCR. (B) Huh-7 and HEP 3B2.1-7 cells were transfected with miR-490-3p mimics or NC mimics for 48 h, and the expression of miR-490-3p was measured using RT-qPCR. (C) Huh-7 and (D) HEP 3B2.1-7 cells were transfected with miR-490-3p mimics or NC mimics for 0, 24, 48 and 72 h. Cell viability was detected using Cell Counting Kit-8 assay. ${ }^{* *} \mathrm{P}<0.01$ vs. NC group. miR, microRNA; HCC, hepatocellular carcinoma; RT-qPCR, reverse transcription-quantitative PCR; NC, negative control; OD, optical density.

Transwell assay. The migration and invasion abilities of Huh-7 and HEP 3B2.1-7 cells were evaluated using 24-well Transwell chambers (Corning, Inc.). Briefly, a total of $1 \times 10^{4}$ cells/well were seeded into the upper chamber containing DMEM without serum. In addition, DMEM supplemented with $10 \%$ FBS was added into the lower chamber to induce cell migration. Following 24-h incubation, migratory or invaded cells on the lower surface of the membranes were fixed and stained with $0.2 \%$ crystal violet at room temperature for $30 \mathrm{~min}$. Photomicrographs of the migratory and invaded cells were captured using a laser confocal microscope (Olympus CX23; Olympus Corporation) and the cell number was counted in five randomly selected fields. For the Transwell invasion assay, the upper chamber was pre-coated with Matrigel (BD Biosciences).

Luciferase activity assay. TargetScan (http://www.targetscan. org/vert_71/) predicted that TMOD3 is potential target of miR-490-3p. Therefore, the putative miR-490-3p binding sequence of wild type (WT) TMOD3 (CCAGGUUA) and a mutant sequence (MT; GGCGGUC) were synthesized and sub-cloned into the luciferase reporter plasmid pMIR (Shanghai GenePharma Co.,Ltd.) to obtain pMIR-WT-TMOD3 and p-MIR-MT-TMOD3 plasmids, respectively. Subsequently, cells were co-transfected with the luciferase plasmids (WT or MT) and miR-490-3p or NC mimics using Lipofectamine 2000. Following 48-h incubation, the luciferase activity in each group was detected using the Dual-Luciferase Reporter Assay System (Promega Corporation) with Renilla luciferase activity serving as endogenous control.

Statistical analysis. Experiments were replicated three times and statistical analyses were conducted using GraphPad Prism 7 software (GraphPad Inc.). Results are presented as mean \pm standard deviation. The data were compared using one-way ANOVA followed by Tukey's test, and $\mathrm{P}<0.05$ was considered to indicate a statistically significant difference.

\section{Results}

Overexpression of miR-490-3p inhibits the proliferation of HCC cells. The expression of miR-490-3p in human hepatocytes and HCC cells was evaluated using RT-qPCR. The data indicate the level of miR-490-3p was significantly lower in HCC cells compared with that in human hepatocytes (Fig. 1A). In order to explore the role of miR-490-3p in HCC, Huh-7 and HEP 3B2.1-7 cells were transfected with miR-490-3p mimics. As shown in Fig. 1B, the expression levels of miR-490-3p were significantly increased in Huh-7 and HEP 3B2.1-7 cells following transfection with miR-490-3p mimics compared with the NC group. Subsequently, a CCK-8 assay was performed to investigate the effect of miR-490-3p on the viability of the HCC cells. The results reveal that miR-490-3p overexpression significantly reduced the viability of Huh-7 and HEP 3B2.1-7 
A

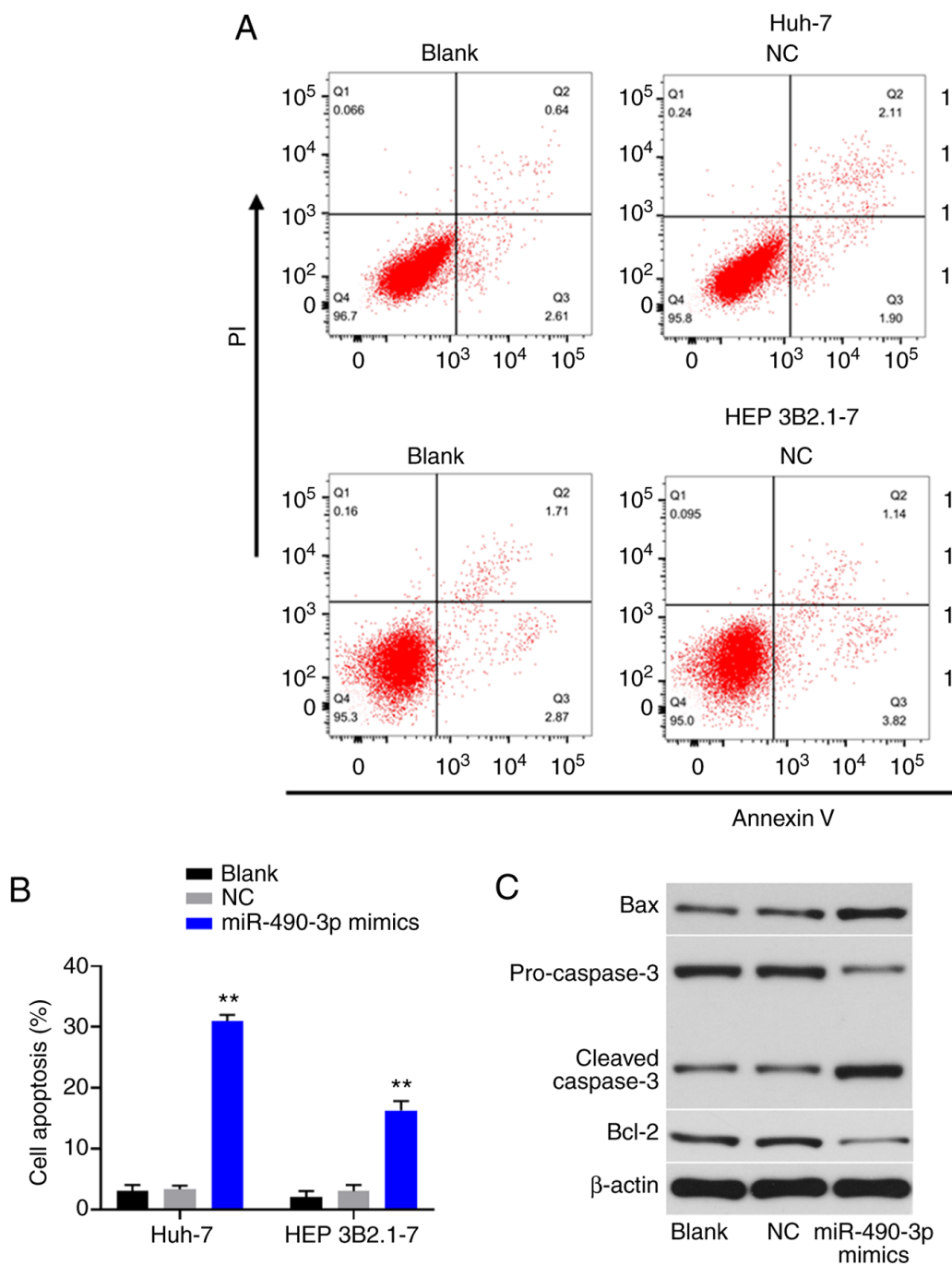

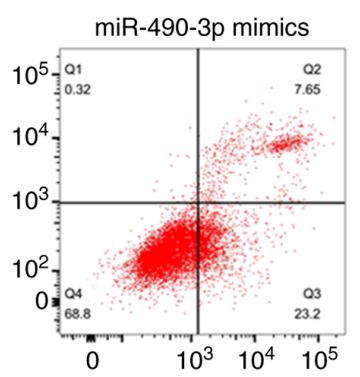

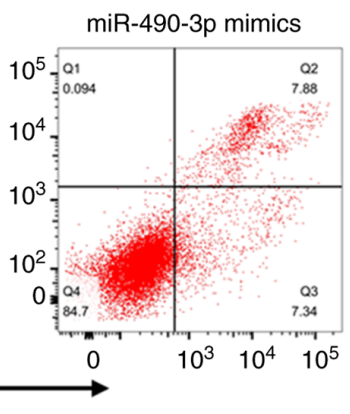

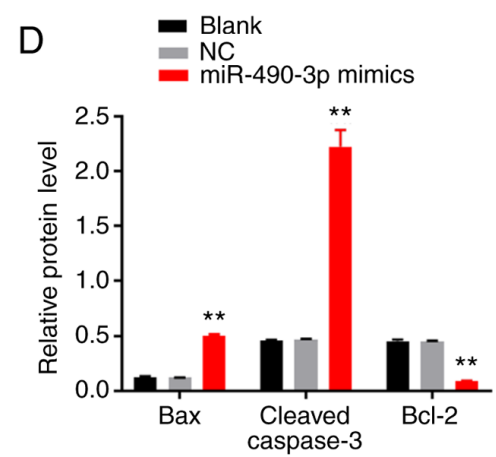

Figure 2. Overexpression of miR-490-3p induces apoptosis of Huh-7 and HEP 3B2.1-7 cells. Huh-7 and HEP 3B2.1-7 cells were transfected with miR-490-3p mimics or NC mimics for $6 \mathrm{~h}$ and then cultured for another $42 \mathrm{~h}$. (A and B) Apoptotic cells were detected with Annexin V-PI double staining and flow cytometric analysis. (A) Representative flow cytometry images and (B) quantified apoptosis data are shown. (C) Protein levels of Bax, pro- and cleaved caspase-3 and Bcl-2 in Huh-7 cells were determined using western blotting. (D) Relative levels of Bax and Bcl-2 were quantified via normalization to $\beta$-actin, and relative levels of cleaved caspase-3 were quantified via normalization to pro-caspase-3. ${ }^{* *} \mathrm{P}<0.01 \mathrm{vs}$. NC group. miR, microRNA; NC, negative control.

cells (Fig. 1C and D). The aforementioned results indicate that the overexpression of miR-490-3p inhibited the proliferation of HCC cells.

Overexpression of $\mathrm{miR}-490-3 \mathrm{p}$ induces $\mathrm{Huh}-7$ and $\mathrm{HEP}$ 3B2.1-7 cell apoptosis. Apoptosis is an important biological process in cancer cells; therefore, the effect of miR-490-3p mimics on the apoptosis of HCC cells was investigated. As shown in Fig. 2A and B, flow cytometric analysis reveal that the apoptosis rates of Huh-7 and HEP 3B2.1-7 cells were significantly increased following transfection with miR-490-3p mimics compared with those in the corresponding NC group (Fig. 2A and B). In addition, western blotting results demonstrate that the levels of the pro-apoptotic proteins Bax and cleaved caspase- 3 were significantly increased, while the expression levels of the anti-apoptotic protein Bcl-2 were significantly decreased in Huh-7 cells following transfection with miR-490-3p mimics compared with the NC group (Fig. 2C and D). These results suggest that the overexpression of miR-490-3p induced the apoptosis of Huh-7 cells.
Overexpression of $m i R-490-3 p$ attenuates the migration and invasion of HCC cells. To investigate the role of miR-490-3p in the migration and invasion of HCC cells, Transwell migration and invasion assays were performed. The results demonstrate that miR-490-3p mimics significantly decreased the migration ability of Huh-7 and HEP 3B2.1-7 HCC cells compared with the respective NC group (Fig. 3A). In addition, the Transwell invasion assay indicated that invasion of the HCC cells was significantly inhibited following transfection with miR-490-3p mimics (Fig. 3B). The data suggest that the overexpression of miR-490-3p attenuated the migration and invasion of HCC cells.

miR-490-3p directly targets TMOD3. To further investigate the mechanisms by which miR-490-3p affects HCC tumorigenesis, TargetScan analysis was performed to identify the potential targets of miR-490-3p. The screening results indicate that TMOD3 is a potential target of miR-490-3p (Fig. 4A). A dual luciferase reporter system assay was then used to confirm the association between miR-490-3p and TMOD3. As shown in Fig. 4B, luciferase activity was significantly reduced in cells 
A

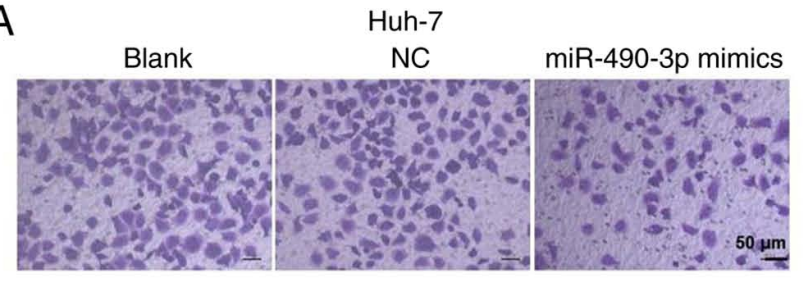

HEP 3B2.1-7
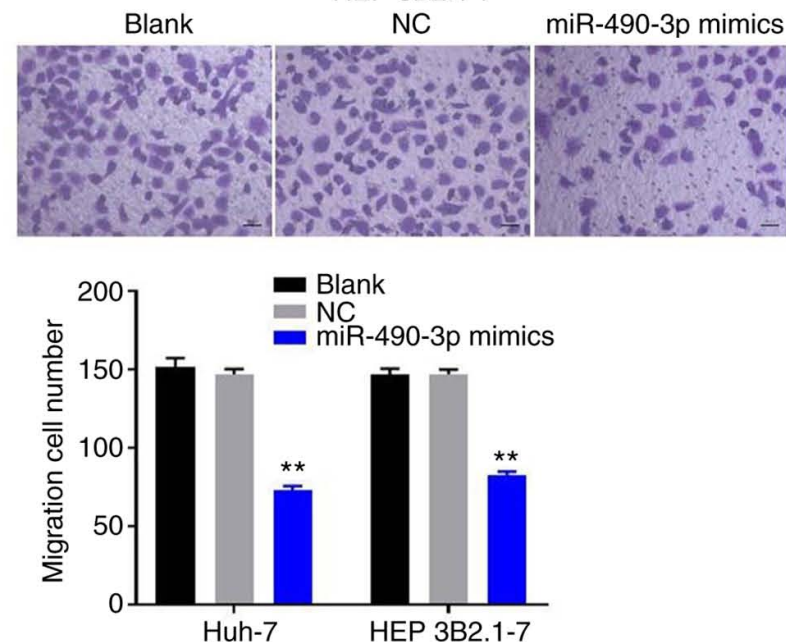

B

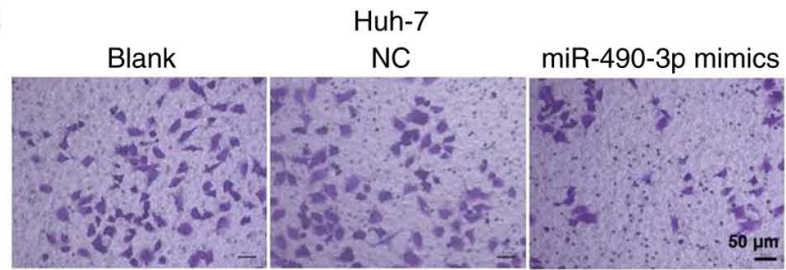

HEP 3B2.1-7
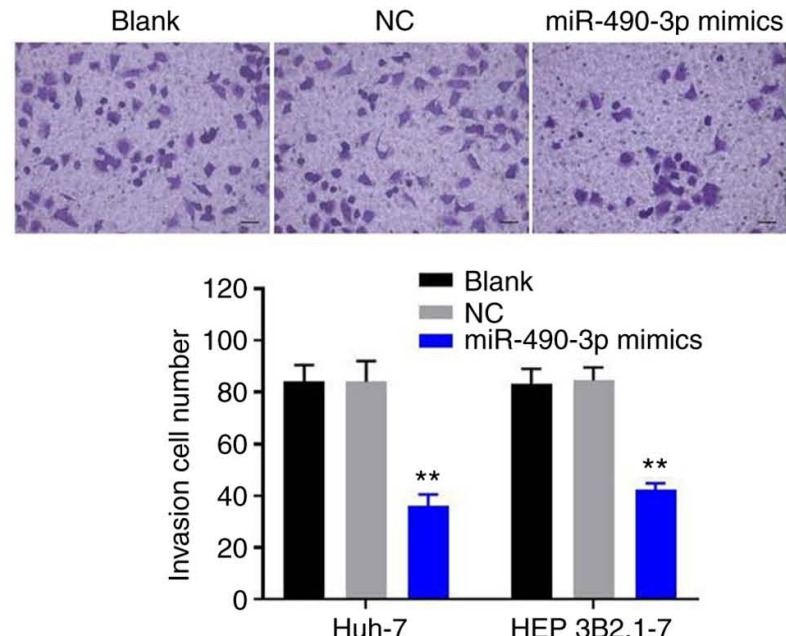

Figure 3. Overexpression of miR-490-3p attenuates the migration and invasion of Huh-7 and HEP 3B2.1-7 cells. Huh-7 and HEP 3B2.1-7 cells were transfected with miR-490-3p mimics or mimics NC for $6 \mathrm{~h}$ and then cultured for another $18 \mathrm{~h}$. (A) Transwell migration assay was used to measure the migration of Huh-7 and HEP 3B2.1-7 cells. (B) Transwell invasion assay was used to measure the invasion of Huh-7 and HEP 3B2.1-7 cells. ${ }^{* *} \mathrm{P}<0.01$ vs. NC group. miR, microRNA; NC, negative control.

\section{A TMOD3 651-658 5'-...AAGUCAUCAGCUUUGCCAGGUUA...-3 (wild type) miR-490-3p 3'-GUCGUACCUCAGGAGGUCCAAC-'5 TMOD3 651-658 5'-...CACAAAAAUUUGAACGGCGGUCA...-3' (Mutant)}

$\mathrm{B}$

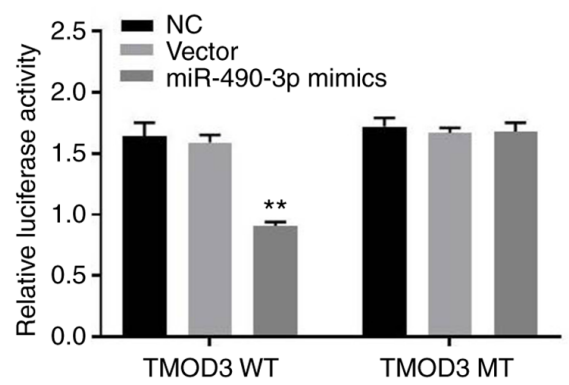

C

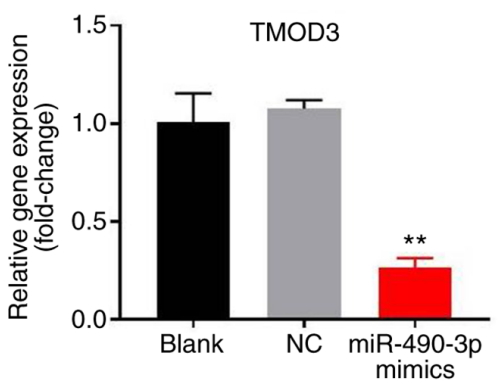

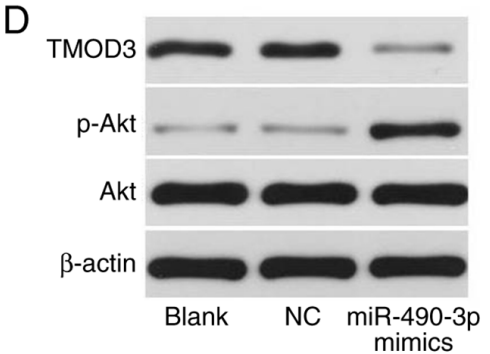
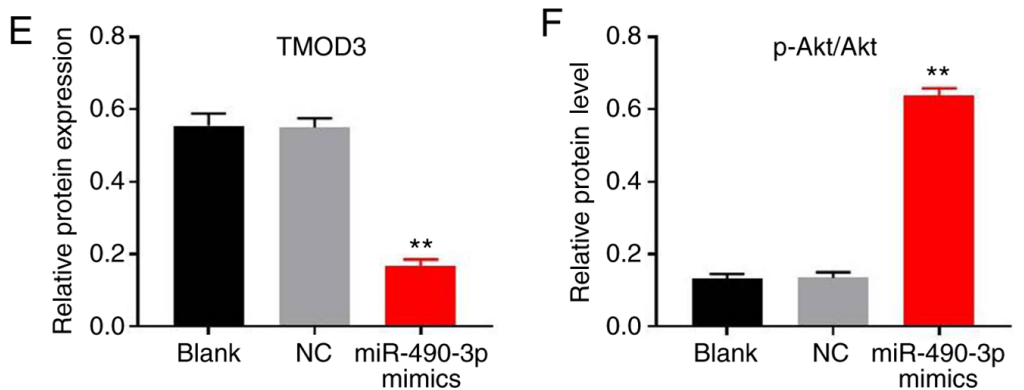

Figure 4. TMOD3 is a direct binding target of miR-490-3p. (A) Sequence of wild-type TMOD3 that is predicted to be targeted by miR-490-3p and the sequence of the mutant TMOD3. (B) Luciferase activity was measured following co-transfection with TMOD3 plasmids and miR-490-3p mimics. (C) TMOD3 levels in Huh-7 cells transfected with the miR-490-3p mimics were detected by reverse transcription-quantitative PCR. (D) TMOD3 expression and p-Akt levels in Huh-7 cells were determined via western blotting. (E) Relative expression of TMOD3 was quantified via normalization to $\beta$-actin. (F) Relative p-Akt levels were quantified via normalization to Akt. ${ }^{* *} \mathrm{P}<0.01$ vs. NC group. TMOD3, tropomodulin 3; miR, microRNA; NC, negative control; WT, wild type; MT, mutant; p, phosphorylated. 

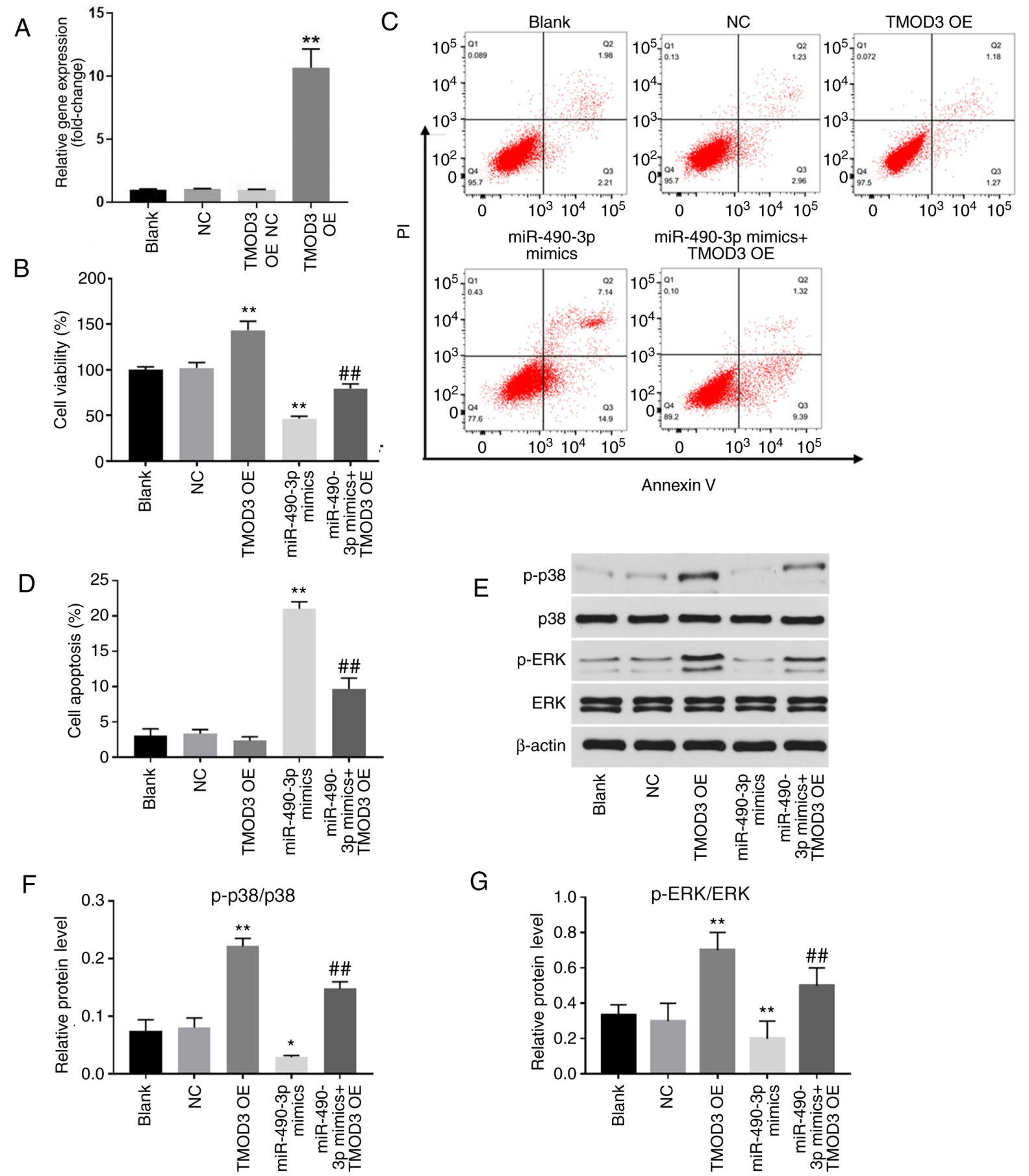

G

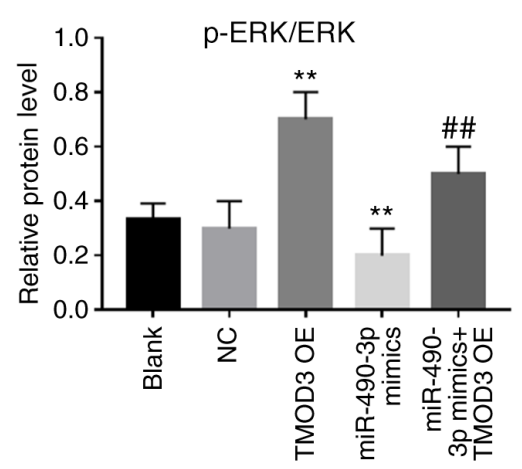

Figure 5. miR-490-3p acts as a tumor suppressor in Huh-7 cells via the negative regulation of TMOD3. Huh-7 cells were transfected with TMOD3-OE or/and miR-490-3p mimics for $72 \mathrm{~h}$. (A) The successful overexpression of TMOD3 in cells following transfection with TMOD3 OE plasmid was confirmed with reverse transcription-quantitative PCR. (B) Cell viability was detected using Cell Counting Kit-8 assay. (C) Apoptotic cells were detected with Annexin V-PI double staining and flow cytometric analysis. (C) Representative flow cytometry images and (D) quantified apoptosis data are shown. (E) Levels of p-p38, p38, p-ERK and ERK in Huh-7 cells were determined using western blotting. ( $F$ and G) Relative levels of p-p38 and p-ERK were quantified via normalization to $\mathrm{p} 38$ and ERK, respectively. ${ }^{*} \mathrm{P}<0.05,{ }^{* *} \mathrm{P}<0.01$ vs. NC group; ${ }^{\# \#} \mathrm{P}<0.01$ vs. miR-490-3p mimics group. miR, microRNA; TMOD3, tropomodulin 3; OE, overexpression; NC, negative control; TMOD3 OE-NC, empty plasmid; p, phosphorylated.

co-transfected with miR-490-3p mimics and TMOD3-WT compared with that in the corresponding NC group. However, miR-490-3p mimics did not affect the luciferase activity of TMOD3-MT. Furthermore, RT-qPCR and western blot assays showed that miR-490-3p mimics significantly downregulated the expression levels of TMOD3 in Huh-7 cells compared with those in the NC group (Fig. 4C-E). Furthermore, the level of phosphorylation of Akt in Huh-7 cells was decreased by
miR-490-3p mimics (Fig. 4D and F). These data confirm that TMOD3 is a direct target of miR-490-3p.

miR-490-3p acts as a tumor suppressor in Huh-7 cells via the negative regulation of TMOD3. Although the aforementioned results verify that TMOD3 is a direct binding target of miR-490-3p, the underlying mechanisms of miR-490-3p in HCC tumorigenesis remain largely unknown. Therefore, 
TMOD3 was overexpressed in Huh-7 cells to further explore the mechanism (Fig. 5A). CCK-8 assay revealed that miR-490-3p mimics significantly reduced the viability of Huh-7 cells, while this effect was markedly attenuated following the overexpression of TMOD3 (Fig. 5B). In addition, miR-490-3p mimics-induced apoptosis was significantly decreased in Huh-7 cells following TMOD3 overexpression (Fig. 5C and D). Furthermore, western blot assay demonstrated that miR-490-3p mimics significantly downregulated the levels of p-p38 and p-ERK in Huh-7 cells, (Fig. 5E-G). Additionally, the miR-490-3p mimics-induced downregulation of p38 and ERK phosphorylation was significantly attenuated by TMOD3 overexpression (Fig. 5E-G). These data indicate that miR-490-3p acted as a tumor suppressor in Huh-7 cells via the negative regulation of TMOD3, p-p38 and p-ERK.

\section{Discussion}

A multitude of miRNAs have already been considered as therapeutic targets for HCC in preclinical models, thus providing a credible bench-to-bedside connection. For example, Gougelet et al (14) showed that miR-34a exhibited an oncogenic role in liver cancer, as its downregulation inhibited the proliferation of hepatocytes. In addition, Pineau et al (15) demonstrated that miR-221 served an important role in hepatocarcinogenesis and its overexpression promoted the proliferation of HCC cells. By contrast, Zheng et al (12) suggested that the expression levels of miR-490-3p were significantly downregulated in HCA and HCC tissues. These findings prompted the further investigation of the role of miR-490-3p in HCC cells in the present study. The results indicate that the upregulation of miR-490-3p inhibited the proliferation and invasion of HCC cells.

It has been reported that miR-490-3p expression is associated with several types of cancer. Tian et al (16) demonstrated that miR-490-3p increased the cisplatin sensitivity of ovarian cancer cells via the negative regulation of ATP binding cassette subfamily $\mathrm{C}$ member 2 expression. In addition, Kang et al (17) revealed an inhibitory effect of miR-490-3p on esophageal squamous cell carcinoma tissues and cells, which proceeded via the targeting of high mobility group A2, while Qu et al (18) found that decreased levels of miR-490-3p were associated with poor clinical outcome in Helicobacter pylori-induced gastric cancer. Furthermore, in another study, upregulated miR-490-3p expression inhibited the growth and invasiveness of colorectal cancer cells by inhibiting the expression of the oncogene voltage dependent anion channel 1 (19). In the present study, the overexpression of miR-490-3p significantly inhibited the proliferation, migration and invasion of HCC cells. Furthermore, the overexpression of miR-490-3p significantly induced apoptosis in HCC cells via upregulation of the protein expression levels of Bax and cleaved caspase-3. Overall, consistent with the aforementioned previous studies, the present data indicate that miR-490-3p may be an important biomarker in HCC.

It is well documented that miRNAs exert their biological functions by negatively regulating the expression of target genes. To investigate the underlying mechanism of miR-490-3p in the progression of HCC, a TargetScan analysis and luciferase reporter assay were performed to predict and identify, respectively, the potential target genes of miR-490-3p. The results indicate that TMOD3 is a target of miR-490-3p. TMOD3, a unique tropomodulin, belongs to the pointed-end capping protein family, which is involved in the regulation of the actin filament structure in diverse cell types (20). It has been reported that TMOD3 negatively regulates endothelial cell migration and serves a crucial role in asymmetric division during oocyte maturation $(21,22)$. Furthermore, Jin et al $(23)$ demonstrated that TMOD3 promotes liver cancer progression via activation of the MAPK/ERK signalling pathway. Consistent with this, Zheng et al (24) confirmed that the EGFR-PI3K-AKT signalling pathway is activated by TMOD3 during hepatocarcinogenesis. Functions of TMOD3 have been observed in cancers other than liver cancer $(25,26)$. In a study conducted by Zhan et al (25), an interaction with TMOD3 was found to be involved in the protumorigenic activity of lysyl oxidase-like 2 in esophageal cancer cells. In another study, conducted by Paul et al (26), TMOD3 was identified as a protein associated with etoposide chemoresistance in non-small cell lung carcinoma cells. To the best of our knowledge, the present study is the first to reveal the association between miR-490-3p overexpression and TMOD3 in HCC cells.

In conclusion, the results of the present study suggested that miR-490-3p inhibits the proliferation and invasion of HCC cells via the downregulation TMOD3. Therefore, miR-490-3p may be a potential biomarker and therapeutic target for the diagnosis and treatment of $\mathrm{HCC}$, respectively.

\section{Acknowledgements}

Not applicable.

\section{Funding}

No funding was received.

\section{Availability of data and materials}

The datasets used and/or analyzed during the current study are available from the corresponding author on reasonable request.

\section{Authors' contributions}

HW, YY, and PG analyzed and interpreted the experimental data, and were major contributors to the development of the first draft of the present manuscript. GY designed the study, analyzed the data, reviewed and approved the final draft of the manuscript prior to submission. All authors approved the final manuscript.

\section{Ethics approval and consent to participate}

Not applicable.

\section{Patient consent for publication}

Not applicable.

\section{Competing interests}

The authors declare that they have no competing interests. 


\section{References}

1. Bray F, Ferlay J, Soerjomataram I, Siegel RL, Torre LA and Jemal A: Global cancer statistics 2018: GLOBOCAN estimates of incidence and mortality worldwide for 36 cancers in 185 countries. CA Cancer J Clin 68: 394-424, 2018.

2. Wallace MC, Preen D, Jeffrey GP and Adams LA: The evolving epidemiology of hepatocellular carcinoma: A global perspective. Expert Rev Gastroenterol Hepatol 9: 765-779, 2015.

3. Blanc JF, Frulio N, Chiche L, Bioulac-Sage P and Balabaud C: Hepatocellular adenoma management: Advances but still a long way to go. Hepat Oncol 2: 171-180, 2015.

4. Omyła-Staszewska J and Deptała A: Effective therapeutic management of hepatocellular carcinoma-on the basis of a clinical case. Contemp Oncol (Pozn) 16: 60-63, 2012.

5. Bartel DP: MicroRNAs: Genomics, biogenesis, mechanism, and function. Cell 116: 281-297, 2004.

6. Rupaimoole R and Slack FJ: MicroRNA therapeutics: Towards a new era for the management of cancer and other diseases. Nat Rev Drug Discov 16: 203-222, 2017.

7. Li L, Yuan L, Luo J, Gao J, Guo J and Xie X: miR-34a inhibits proliferation and migration of breast cancer through down-regulation of Bcl-2 and SIRT1. Clin Exp Med 13: 109-117, 2013.

8. Yan LX, Huang XF, Shao Q, Huang MY, Deng L, Wu QL, Zeng YX and Shao JY: MicroRNA miR-21 overexpression in human breast cancer is associated with advanced clinical stage, lymph node metastasis and patient poor prognosis. RNA 14: 2348-2360, 2008.

9. Zhu Z, Zhang X, Wang $\mathrm{G}$ and Zheng $\mathrm{H}$ : Role of microRNAs in hepatocellular carcinoma. Hepat Mon 14: e18672, 2014.

10. Garofalo M, Di Leva G, Romano G, Nuovo G, Suh SS, Ngankeu A Taccioli C, Pichiorri F, Alder H, Secchiero P, et al: miR-221\&222 regulate TRAIL resistance and enhance tumorigenicity through PTEN and TIMP3 downregulation. Cancer Cell 16: 498-509, 2009.

11. le Sage C, Nagel R, Egan DA, Schrier M, Mesman E, Mangiola A, Anile C, Maira G, Mercatelli N, Ciafrè SA, et al: Regulation of the p27(Kip1) tumor suppressor by miR-221 and miR-222 promotes cancer cell proliferation. EMBO J 26: 3699-3708, 2007.

12. Zheng J, Sadot E, Vigidal JA, Klimstra DS, Balachandran VP, Kingham TP, Allen PJ, D'Angelica MI, DeMatteo RP, Jarnagin WR and Ventura A: Characterization of hepatocellular adenoma and carcinoma using microRNA profiling and targeted gene sequencing. PLoS One 13: e0200776, 2018.

13. Livak KJ and Schmittgen TD: Analysis of relative gene expression data using real-time quantitative PCR and the 2(-Delta Delta C(T)) method. Methods 25: 402-408, 2001

14. Gougelet A, Sartor C, Bachelot L, Godard C, Marchiol C, Renault G, Tores F, Nitschke P, Cavard C, Terris B, et al: Antitumour activity of an inhibitor of miR-34a in liver cancer with $\beta$-catenin-mutations. Gut 65: 1024-1034, 2016.
15. Pineau P, Volinia S, McJunkin K, Marchio A, Battiston C, Terris B, Mazzaferro V, Lowe SW, Croce CM and Dejean A: miR-221 overexpression contributes to liver tumorigenesis. Proc Natl Acad Sci USA 107: 264-269, 2010.

16. Tian J, Xu YY, Li L and Hao Q: miR-490-3p sensitizes ovarian cancer cells to cisplatin by directly targeting ABCC2. Am J Transl Res 9: 1127-1138, 2017.

17. Kang NN, Ge SL, Zhang RQ, Huang YL, Liu SD and Wu KM miR-490-3p inhibited the proliferation and metastasis of esophageal squamous cell carcinoma by targeting HMGA2. Eur Rev Med Pharmacol Sci 22: 8298-8305, 2018.

18. Qu M, Li L and Zheng WC: Reduced miR-490-3p expression is associated with poor prognosis of Helicobacter pylori induced gastric cancer. Eur Rev Med Pharmacol Sci 21: 3384-3388, 2017.

19. Liu X, He B, Xu T, Pan Y,Hu X, Chen X and Wang S: miR-490-3p functions as a tumor suppressor by inhibiting oncogene VDAC1 expression in colorectal cancer. J Cancer 9: 1218-1230, 2018.

20. Yamashiro S, Gokhin DS, Kimura S, Nowak RB and Fowler VM: Tropomodulins: Pointed-end capping proteins that regulate actin filament architecture in diverse cell types. Cytoskeleton (Hoboken) 69: 337-370, 2012.

21. Fischer RS, Fritz-Six KL and Fowler VM: Pointed-end capping by tropomodulin 3 negatively regulates endothelial cell motility. J Cell Biol 161: 371-380, 2003.

22. Jo YJ, Jang WI, Kim NH and Namgoong S: Tropomodulin-3 is essential in asymmetric division during mouse oocyte maturation. Sci Rep 6: 29204, 2016.

23. Jin C, Chen Z, Shi W and Lian Q: Tropomodulin 3 promotes liver cancer progression by activating the MAPK/ERK signaling pathway. Oncol Rep 41: 3060-3068, 2019.

24. Zheng H, Yang Y, Hong YG, Wang MC, Yuan SX, Wang ZG, Bi FR, Hao LQ, Yan HL and Zhou WP: Tropomodulin 3 modulates EGFR-PI3K-AKT signaling to drive hepatocellular carcinoma metastasis. Mol Carcinog 58: 1897-1907, 2019.

25. Zhan XH, Jiao JW, Zhang HF, Xu XE, He JZ, Li RL, Zou HY, Wu ZY, Wang SH, Wu JY, et al: LOXL2 upregulates phosphorylation of ezrin to promote cytoskeletal reorganization and tumor cell invasion. Cancer Res 79: 4951-4964, 2019.

26. PaulD, Chanukuppa V, Reddy PJ, Taunk K, Adhav R, Srivastava S, Santra MK and Rapole S: Global proteomic profiling identifies etoposide chemoresistance markers in non-small cell lung carcinoma. J Proteomics 138: 95-105, 2016.

(7) $\odot$ This work is licensed under a Creative Commons Attribution-NonCommercial-NoDerivatives 4.0 International (CC BY-NC-ND 4.0) License. 